\title{
Nano-droplets Deposited in Microarrays by Femtosecond Ti:Sapphire Laser-Induced Forward Transfer
}

\author{
David P. Banks, Christos Grivas, John D. Mills, \\ and Robert W. Eason \\ Optoelectronics Research Centre (O.R.C), University of Southampton, Southampton \\ SO17 1BJ, United Kingdom
}

loanna Zergioti

National Technical University of Athens, Physics Department, 15780 Zografou, Athens, Greece

\begin{abstract}
We present the deposition of nanoscale droplets of Cr using femtosecond Ti:Sapphire LaserInduced Forward Transfer. Deposits around $300 \mathrm{~nm}$ in diameter, significantly smaller than any previously reported, are obtained from a $30 \mathrm{~nm}$ thick source film. Deposit size, morphology, and adhesion to a receiver substrate as functions of applied laser fluence are investigated. We show that deposits can be obtained from previously irradiated areas of the source material film with negligible loss of deposition quality, allowing sub-spot size period microarrays to be produced without the need to move the source film.
\end{abstract}


The Laser-Induced Forward Transfer (LIFT) technique exists as a method for the directwriting of a wide variety of materials, currently with minimum achievable resolution around 1 $\mu \mathrm{m}{ }^{1}$ It is of particular interest due to the ability to pattern material in air and at room temperature onto virtually any substrate. ${ }^{2}$ In recent years, significant study has been directed towards extending the range of materials that can be deposited using LIFT; metals, ${ }^{3}$ oxides, ${ }^{2}$ superconductors, ${ }^{4} \mathrm{DNA},{ }^{5}$ proteins, ${ }^{6}$ fungal spores, ${ }^{7}$ polycrystalline- $\mathrm{Si},{ }^{8}$ and various important electronic and sensing materials ${ }^{9}$ have all been transferred. In contrast, efforts to reduce the minimum achievable deposition dimensions have received comparatively little attention. LIFT using nanosecond pulsed lasers (ns-LIFT) typically produces depositions which at best reproduce the shape and size of the laser focal spot. ${ }^{10}$ Femtosecond-LIFT (fs-LIFT) using a UV excimer laser has been shown to be capable of sub-spot size depositions with diameters around $0.5 \mu \mathrm{m} .{ }^{3}$ Recently it was demonstrated that ns-LIFT could also be used to produce sub-spot size deposits by carefully controlling the laser fluence just above the threshold for material transfer. $^{11}$

In LIFT, a thin film of the material to be deposited (the "source film") is coated onto one face of a transparent substrate (the "carrier"), and brought into close contact with the substrate to be deposited onto (the "receiver"). A single laser pulse is then focussed through the carrier onto the carrier-film interface, where it is absorbed in a shallow layer of the film (Fig 1a). Conventionally LIFT then occurs by vaporisation of film material at the constrained interface, with the resultant pressure build-up propelling film material to the receiver. However, this is the case only for thicker films and high laser fluence, for thin films and fluence just above the threshold for material transfer, it is possible for LIFT to occur solely by melt-through of the source film (Fig 1b). With precise control of the laser fluence, only the centre of the melt front reaches the free surface of the film (Fig 1c), allowing molten material under pressure to expand 
through an unconstrained, sub-spot size region. With ns-LIFT this process has been shown to facilitate sub-laser spot size printing. ${ }^{11}$

In this letter we demonstrate that the same process can occur with fs-LIFT, and also that the restriction of lateral heat diffusion in the film by the ultrashort timescales involved can result in significantly smaller depositions than previously reported. ${ }^{3,11}$ The clear advantage of such fsLIFT processing lies in the intrinsically high repetition rates from such laser systems, leading to the potential for large area direct-writing. Although the results we present here involve a 1 $\mathrm{kHz}$ laser pulse train, the technique requires only hundreds of $\mathrm{nJ}$ pulse energies, which are achievable with $\mathrm{MHz}$ repetition rate femtosecond lasers.

Single pulses from a Ti:Sapphire laser $(800 \mathrm{~nm}, 110 \mathrm{fs})$ were used to carry out fs-LIFT experiments. The centre of the $4 \mathrm{~mm}$ diameter (full width, half maximum) Gaussian laser output illuminated a $120 \mu \mathrm{m}$ diameter circular aperture to give an approximately uniform profile. The aperture was imaged onto the carrier-film interface using a 100x microscope objective (N.A $=0.8$, Nikon), resulting in a spot diameter of approximately $4 \mu \mathrm{m}$ at the film surface, as estimated by the laser-induced damage area on the film. Shot-to-shot pulse energy was determined by measuring a fraction $(\sim 8 \%)$ of the incident energy with a fast photodiode allowing laser fluence at the film, $\mathrm{J}$, to be calculated based on the pulse energy and the spot size.

Borosilicate plano-concave lenses of $1 \mathrm{~m}$ focal length (i.e. $\sim 0.5 \mathrm{~m}$ radius of curvature) were used as carriers; these allowed for the accurate determination of carrier-receiver separation, $\mathrm{z}$, when the carrier was rested, concave face down, on the receiver. In all experiments, z was kept at or below $1 \mu \mathrm{m}$. A $30 \mathrm{~nm}$ thick film of $\mathrm{Cr}$ was evaporated onto the concave face of a carrier lens for the source film. Silicon wafers were used as receivers. Samples were mounted on a computer-controlled, 2-axis translation stage to allow a new area 
of the film to be exposed to each laser pulse. All experiments were performed in air at room temperature.

A thorough fluence scan was conducted to identify the threshold for $\mathrm{Cr}$ transfer, $\mathrm{J}_{\mathrm{TH}}$, which was found to be $\mathrm{J}_{\mathrm{TH}}=325 \pm 5 \mathrm{~mJ} / \mathrm{cm}^{2}$. Fig. 2 shows a typical SEM picture of one of the smallest deposits, produced with $\mathrm{J} \approx \mathrm{J}_{\mathrm{TH}}$. The deposits were extremely circular (inset to Fig. 2) and around 300-350 $\mathrm{nm}$ in diameter, significantly below the laser wavelength and an order of magnitude less than the spot size. A domed profile was observed with a peak height of approximately 50-100 nm, significantly greater than the source film thickness.

Deposits such as that shown in Fig. 2 are believed to have arisen from a nano-droplet of molten material growing on the free surface of the source film. The droplet left the film surface and was transferred to the receiver if it grew sufficiently before resolidification of the film. The origin of such droplets has been attributed to hydrodynamic flow during the rapid expansion and cooling of molten material when the melt front in the source film reaches the unconstrained surface. ${ }^{11}$ The speed with which a droplet impacted on the receiver and the mass of film material transferred would be expected to determine the size and morphology of the resulting deposit. These parameters depend on the temperature of the source film upon droplet release, which itself is a function of the laser fluence. The effect of laser fluence on the resultant depositions is shown in Fig. 3. The sharp material transfer threshold is at $320-325$ $\mathrm{mJ} / \mathrm{cm}^{2}$ where depositions appeared for the first time. Deposition properties were observed then to be relatively independent of fluence up to around $345 \mathrm{~mJ} / \mathrm{cm}^{2}$, with reproducible diameters of 300-400 $\mathrm{nm}$ and the same domed profile shown in Fig. 2.

At $345 \mathrm{~mJ} / \mathrm{cm}^{2}$ a second fluence threshold can be seen, above which the deposit diameters began to increase with applied fluence, and there was a larger spread in the obtained sizes. The increase in diameter may be attributed to greater impact momentum of droplets on the receiver, and the lower surface tension of larger mass droplets. This threshold possibly was due to the 
onset of significant evaporation at the carrier-film interface. Depositions produced with fluence greater than this threshold typically possessed diameters from $300-500 \mathrm{~nm}$, although a minority with significantly larger diameters, up to $\sim 800 \mathrm{~nm}$, were also obtained. We suggest that there were two competing processes in this fluence regime, one being the melt-though LIFT seen at lower fluence that resulted in the smaller depositions, and the other an explosive, and hence more violent, process driven by vaporised film material that resulted in the larger depositions. Which process ultimately affected material transfer would have been highly sensitive to any weak points in the evaporated film, the resultant weakness of which would render those areas more susceptible to explosive transfer. All these deposits also exhibited the interesting feature of a central depressed region, the diameter and depth of which increased with applied fluence (Fig 3); again this feature can be attributed to greater momentum of the droplets during transfer.

Above $360 \mathrm{~mJ} / \mathrm{cm}^{2}$, the smallest deposits were no longer obtained, instead most depositions were around 500-700 $\mathrm{nm}$ in diameter. A possible reason for this would be material transfer becoming almost exclusively vaporisation-driven. The morphology of these deposits was of a thin, flat, and circular central region surrounded by a raised rim (Fig 3).

At around $380 \mathrm{~mJ} / \mathrm{cm}^{2}$ another fluence threshold, $\mathrm{J}_{\mathrm{M}}$, was observed, above which multiple deposits were obtained per single laser shot. This phenomenon we attribute to phase explosion $^{12}$ occurring in the source film. Phase explosion involves the nucleation of gas bubbles throughout a superheated liquid, the number of bubbles being an exponential function of liquid temperature. ${ }^{12}$ It is believed that each of the multiple deposits was the result of a gas bubble gaining sufficient internal pressure to propel a piece of the superheated liquid film to the receiver. The number of deposits produced per laser shot is plotted as a function of fluence in the inset of Fig. 3. It can be seen that the number of deposits increased exponentially with 
fluence, supporting the phase explosion hypothesis. Throughout the fluence scan, deposit adhesion to the receiver was seen to improve with increasing applied laser fluence.

Having produced sub-spot (and sub-wavelength) size depositions, the next question was how closely they could be spaced on the receiver. It would always be possible to move carrier and receiver independently to place deposits arbitrarily close, but this would introduce undesirable extra complexity into the printing technique. To investigate how closely successive deposits could be positioned by only moving the entire LIFT apparatus relative to the laser, lines of deposits were produced by linearly scanning the $x-y$ stage perpendicular to the laser beam. A typical result is shown in Fig. 4. The applied fluence was $\sim 350 \mathrm{~mJ} / \mathrm{cm}^{2}$, the stage was scanned at $1400 \mu \mathrm{m} / \mathrm{sec}$, and the laser repetition rate was $1 \mathrm{kHz}$, resulting in $1.4 \mu \mathrm{m}$ between exposures, well below the $\sim 2 \mu \mathrm{m}$ spot radius. The deposits had the same circular shape and 400-600 nm diameters as deposits produced at the same fluence with no overlap between successive pulses, and any increase in surrounding debris was negligible. This result demonstrates that sub-spot size spacing of deposits with minimal loss of printing quality is achievable with fs-LIFT.

To further investigate micron spacing of deposits on the receiver, 2-dimensional microarrays of various periodicities were produced. A typical array produced using $\sim 340$ $\mathrm{mJ} / \mathrm{cm}^{2}$ and a $3 \mu \mathrm{m}$ period is shown in Fig. 5a. There was a marginal increase in the amount of debris around deposits which may have been caused by areas of the film seeing as many as 4 pulses from successive exposures. It should be noted that there was some irregularity in the positioning of depositions in the microarray (Fig. 5a). However these irregularities were also seen in the corresponding damage to the source film (Fig. 5b) indicating that the problem is external to the LIFT technique, most probably due to one or both of translation stage inconsistencies and single-shot beam pointing instabilities. 
In conclusion, we have shown that fs-LIFT is capable of nano-patterning an order of magnitude smaller than the printing laser spot size. Such deposits are obtained over a relatively narrow fluence range just above the material transfer threshold. The diameters of deposits and adhesion to a receiver substrate have been shown to scale with the applied laser fluence, with diameter increasing and adhesion improving with increasing fluence. We have also shown that the laser spot size does not place a fundamental limit on how close successive depositions can be obtained by depositing sub-spot size period lines and microarrays. This direct-write, high repetition rate process is extremely promising for further demonstrations of micron-scale periodic surface patterning, for optoelectronics applications.

Acknowledgements: The authors are grateful to the Engineering and Physical Sciences Research Council, UK for research funding under grant no. EP/C515668/1. 


\section{REFERENCES}

J. Bohandy, B. F. Kim, and F. J. Adrian, J. Appl. Phys. 60, 1538 (1986).

2 S. Mailis, I. Zergioti, G. Koundourakis, A. Ikiades, A. Patentalaki, , P.

Papakonstantinou, N. A. Vainos, and C. Fotakis, Appl. Opt. 38(11), 2301 (1999).

3 P. Papakonstantinou, N. A. Vainos, and C. Fotakis, Appl. Surf. Sci. 151, 159 (1999).

4 E. Fogarassy, C. Fuchs, F. Kerherve, G. Hauchecorne, and J. Perriere, J. Appl. Phys. 66(1), 457 (1989)

5 P. Serra, M. Colina, J. M. Fernández-Pradas, L. Sevilla, and J. L. Morenza, Appl. Phys. Lett. 85(9), 1639 (2004).

6 P. Serra, J. M. Fernández-Pradas, F. X. Berthet, M. Colina, J. Elvira, and J. L. Morenza, Appl. Phys. A 79, 949 (2004).

7 B. Hopp, T. Smausz, Zs. Antal, N. Kresz, Zs. Bor, and D. Chrisey, J. Appl. Phys. 96(6), 3478 (2004).

8 D. Toet, M. O. Thompson, P. M. Smith, and T. W. Sigmon, Appl. Phys. Lett. 74(15), 2170 (1999).

9 H. Kim, G. P. Kushto, C. B. Arnold, Z. H. Kafafi, and A. Piqué, Appl. Phys. Lett. 85(3), 464 (2004).

10 H. Yamada, T. Sano, T. Nakayama, and I. Miyamoto, Appl. Surf. Sci. 197-198, 411 (2002).

11 D. A. Willis and V. Grosu, Appl. Phys. Lett. 86, 244103-1 (2005).

12 K. H. Song and X. Xu, Appl. Surf. Sci. 127, 111 (1998.). 


\section{FIGURE CAPTIONS}

FIGURE 1: Illustration of temperature-gradient driven heat flow in the source film that allows sub-spot size droplet formation during LIFT.

FIGURE 2: $\quad$ SEM image of a deposit produced using $\mathrm{J} \approx 325 \mathrm{~mJ} / \mathrm{cm}^{2}$. Image was taken at an angle of $45^{\circ}$ to the receiver surface normal. Inset: same deposit from directly above (not to scale).

FIGURE 3: Graph of deposition diameter as a function of applied laser fluence, including SEM images of indicated deposits. Inset: Plot of the number of independent deposition events per laser pulse as function of fluence.

FIGURE 4: SEM image of a line of depositions produced using $\sim 350 \mathrm{~mJ} / \mathrm{cm}^{2}$ with separation of $1.4 \mu \mathrm{m}$ between successive exposures.

FIGURE 5: (a) SEM image of $3 \mu \mathrm{m}$ period array and (b) optical microscope image of corresponding film damage from below. N.B. (b) has been reflected such that holes correspond to same position as deposits in (a). 
Figure 1

Top-hat pulse incident

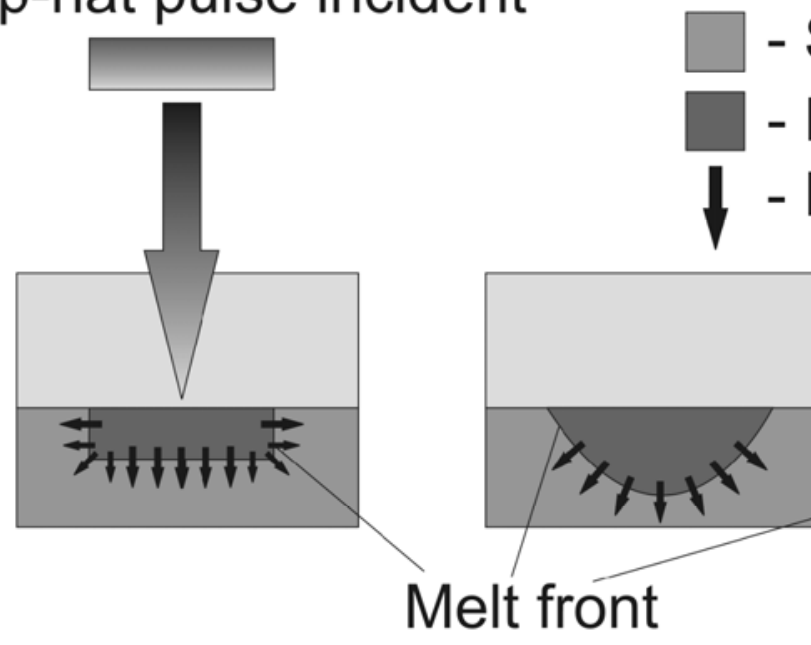

- Transparent carrier

- Source film

- Molten film material

- Direction of heat flow

Molten material expands
a) $t=t_{0}$
b) $t_{1}>t_{0}$
c) $t_{2}>t_{1}$

Figure 2

\section{$330 \mathrm{~nm}$}

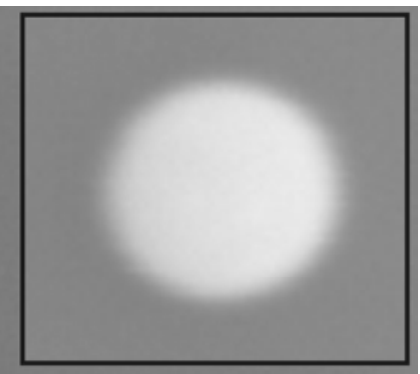


Figure 3

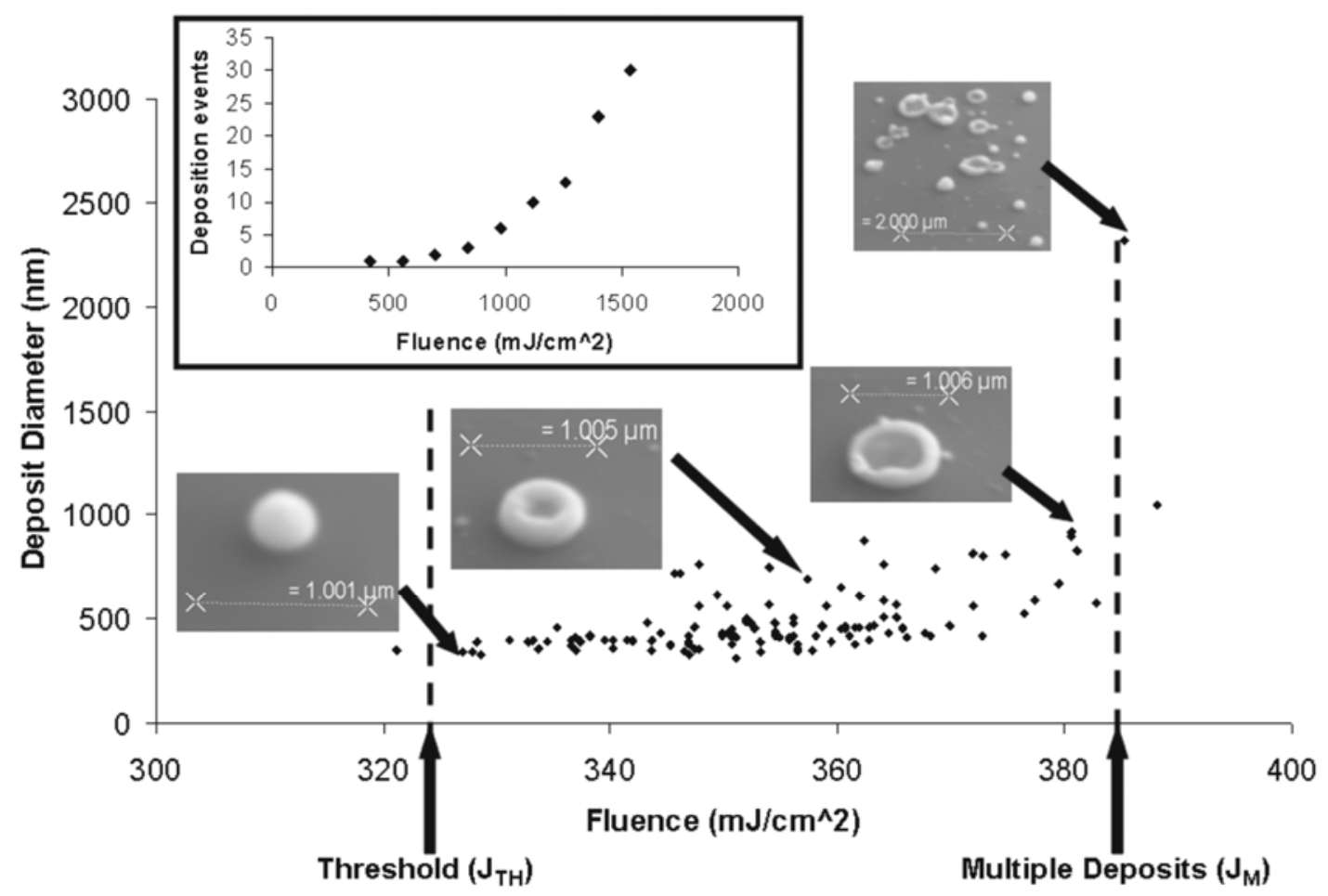

Figure 4

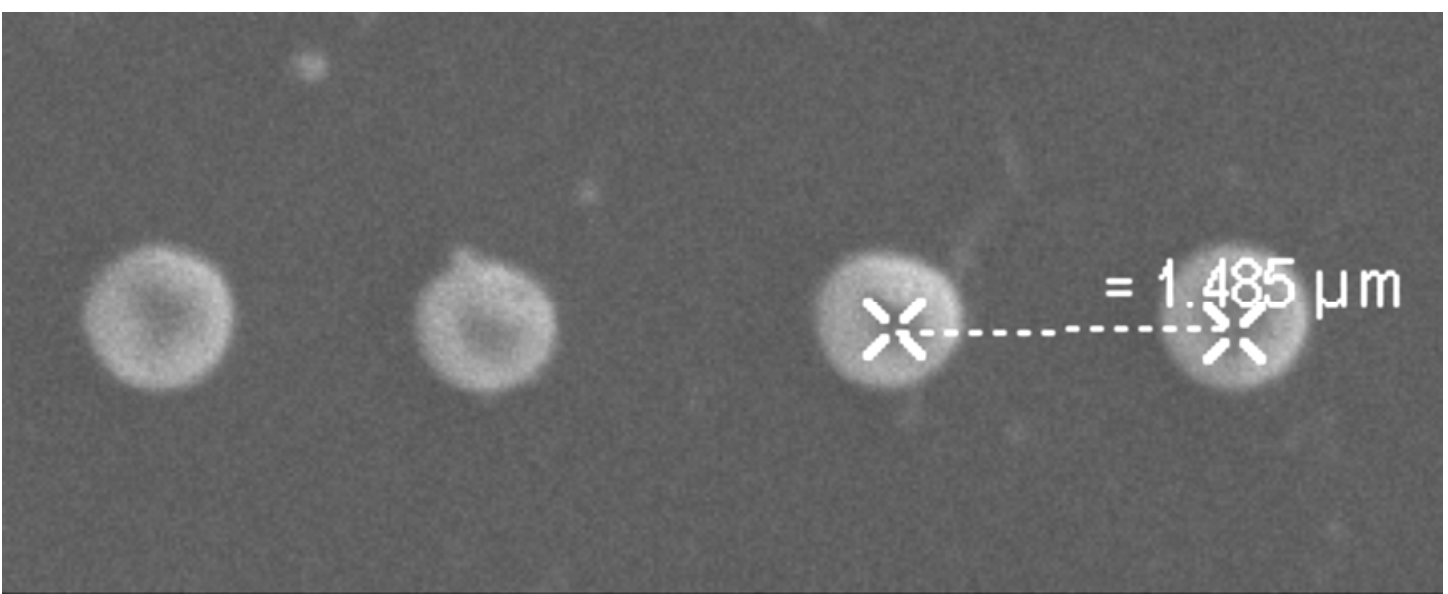


Figure 5
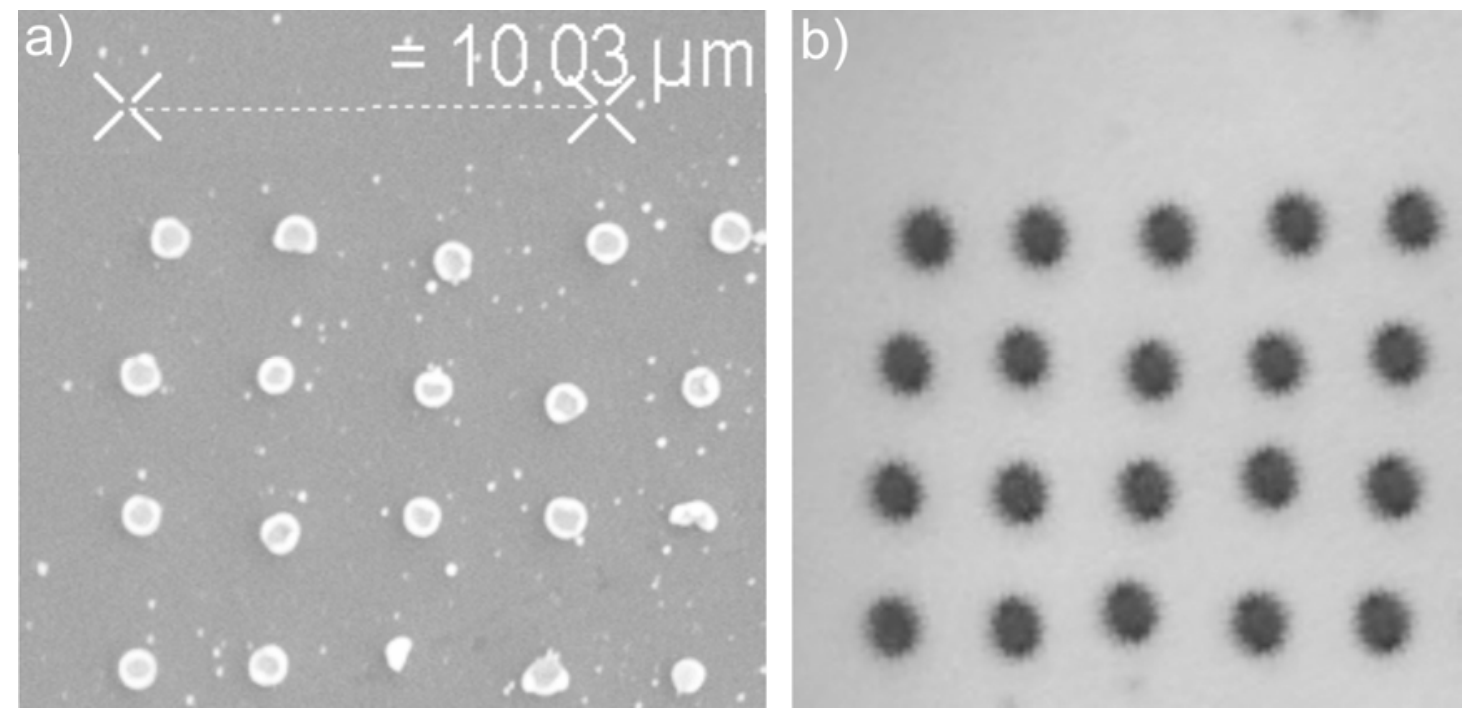